\title{
Extension of the Genomic Conceptual Model to integrate Genome-Wide Association Studies
}

\author{
Federico Comolli \\ Department of Electronics, Information and Bioengineering \\ Politecnico di Milano, Milan, Italy
}

\begin{abstract}
The first human genome has been sequenced at the turn of the year 2000. Since then, modern biology has made great progresses, also thanks to the introduction of Next-generation sequencing in the mid2000s. The growing availability of genomic data led to the birth of tertiary analysis, concerning sense-making and extraction of useful biological information. To deal with data heterogeneity, in the last decade many tools have been introduced to achieve genomic data integration: among them, the Genomic Conceptual Model (GCM) and the META-BASE architecture. The latter one allows to map data from many projects into the GCM through an integration pipeline.

In this work, we proposed an extension of the GCM to integrate two additional sources into the META-BASE architecture, namely: GWAS Catalog (curated by the NHGRI and EBI institutes) and FinnGen (curated by the University of Helsinki). These two sources host GenomeWide Association Studies (GWAS), useful for explaining the connection between genome variations of single nucleotides and particular traits. They are organized according to different data models but share the same data semantics. As a result of our integration efforts, we enable the interoperable use and querying of GWAS datasets with several other genomic datasets (including TCGA, ENCODE, Roadmap Epigenomics, 1000 Genomes Project, and GENCODE).
\end{abstract}

Keywords: Data integration $\cdot$ Genomic Datasets $\cdot$ Bioinformatics $\cdot$ Multiomics Studies · GWAS

\section{Introduction}

Since the mid-2000s, thanks to the introduction of Next-generation sequencing 17, a whole human DNA sequence can be read in a short time and in a cheap way. After being sequenced, the so-called tertiary analysis [16] is performed, dealing with sense-making of the huge amount of data produced by the previous analysis. Big amount of data collected by different consortia need to be integrated to allow scientists to extract information useful to understand how life is orchestrated by the DNA and how the sequence of nucleotides affects diseases or phenotype. Data produced in the context of different projects have different formats, resulting into an obstacle for data interoperability required by the tertiary analysis. 
A big effort to cope with genomic data heterogeneity has been performed by the GeCo project of Politecnico di Milano developing a conceptual model (the Genomic Conceptual Model [2]), a query language (the GenoMetric Query Language [10]) and a pipeline to integrate genomic data from multiple sources (the META-BASE architecture [1]). One of the purposes of the GeCo project has been to create an integrated genomic repository that collects data from major consortia around the world (e.g., 1000 Genomes [19, Cistrome 24], ENCODE 20], GENCODE [5, Roadmap Epigenomics [8], and TCGA [23]).

In this article, we presented the modelling efforts spent to integrate into the META-BASE architecture a new class of studies called Genome-Wide Association Studies (GWAS). They involve testing genetic variants across the genomes of many individuals to identify genotype-phenotype associations. By comparing groups of people affected by a disease or trait (cases) and without it (controls), the outcomes of these studies comprise the more frequent nucleotides in the cases group against the controls. The difference of GWAS from other studies is the focus of the analysis: single nucleotide polymorphisms (SNPs) for GWAS, whole portions of genome or DNA features for other omics studies. GWAS have revolutionized the field of complex disease genetics over the past decade, providing numerous compelling associations for human complex traits and diseases [18. Examples of GWAS are 21], which identified 103 SNPs associated to "schizophrenia", and 7, which found 333 SNPs associated to "multiple sclerosis". This work focuses on two GWAS repositories, namely the GWAS Catalog [3] and the FinnGen Project [4].

Integrating multiple omic repositories (i.e., genomic, proteomic and transcriptomic) into the GCM serves to improve the knowledge about the molecular function and disease etiology. Multi-omic studies combine different biological entities to find novel associations between them, paving the road for disease treatments and prevention. The work in this paper focuses on the integration made on metadata describing experiments, rather than on the genomic region attributes, whose transformation is trivial (see [9]).

\section{Related Works}

Due to the ongoing increase of genomic data, the management techniques for large data can be applied to address the heterogeneity and complexity of the biological field. Many works exploit the conceptual modeling to capture the diverse biological objects and to interpret their relationships (see [22|12|13|15|11|14]). The objective of the cited works is to support biologists to extract insights from raw genomic data. The Genomic Conceptual Model [2, whose extension is introduced in this article, goes further the description and data organization of complex biological integrated repositories; it is an architecture driving the integration of new genomic repositories. The work presented in this article exemplifies how the architecture can be exploited to integrate new datasets, mapping them to a shared conceptual model. 


\section{Background}

A shared conceptual schema, the Genomic Conceptual Model (GCM), has been introduced to describe semantically heterogeneous data. Multiple genomic sources have been mapped over the GCM following the META-BASE pipeline, by extracting and transforming the source-specific metadata.

The GCM is an entity-relationship model used to gather metadata of heterogeneous genomic data sources. It is organized as a star-schema centered on the Item entity from which depart four sub-schemata (or views), recalling a classic star-schema organization that is typical of data warehouses; they respectively describe biological, technological, management and extraction aspects (more thoroughly described in [2]):

- Central Entity: it represents an elementary experimental file of genomic regions and their attributes. Files are typically used by biologists for data extraction, analysis and visualization operations.

- Biological View: it consists of the chain of entities Item-Replicate-BiosampleDonor, representing the biological elements that contribute to the Item production. The Donor represents an individual (characterized by Age, Gender and Ethnicity) or strain of a specific organism (Species) from which the biological material was derived or the cell line was established.

- Technology View: it describes the technology used to produce the data Item. An Item is associated by means of a one-to-many relationship with a given ExperimentType.

- Management View: it consists of the chain of entities Item-Case-Project describing the organizational process for the production of items.

- Extraction View: it includes the entity Dataset, used to describe common properties of homogeneous items.

\section{Data Design}

Samples of the typical integrated sources are assigned to single individuals; for each biological sample we can retrieve the information about the donor(s) who provided it. Instead, Genome-Wide Association Studies are based on cohorts of patients, so the considered granularity is coarser w.r.t. already integrated datasets. For each GWAS sample we know the cohort size and limited ancestral information, while detailed information about each single component of the cohort is not available. For this reason, in order to include the two sources GWAS Catalog and FinnGen into the META-BASE repository, we have extended the GCM introducing the GWAS View, to meet the constraints of the considered class of studies.

Figure 1 illustrates the extended GCM with two added entities, i.e., Cohort and Ancestry, belonging to the GWAS View. In the following, we describe this novel view for including GWAS samples.

Entity Item. It is the central entity of the GCM and it is shared between all its views. A GWAS Item contains all the SNPs associated with the phenotype under 


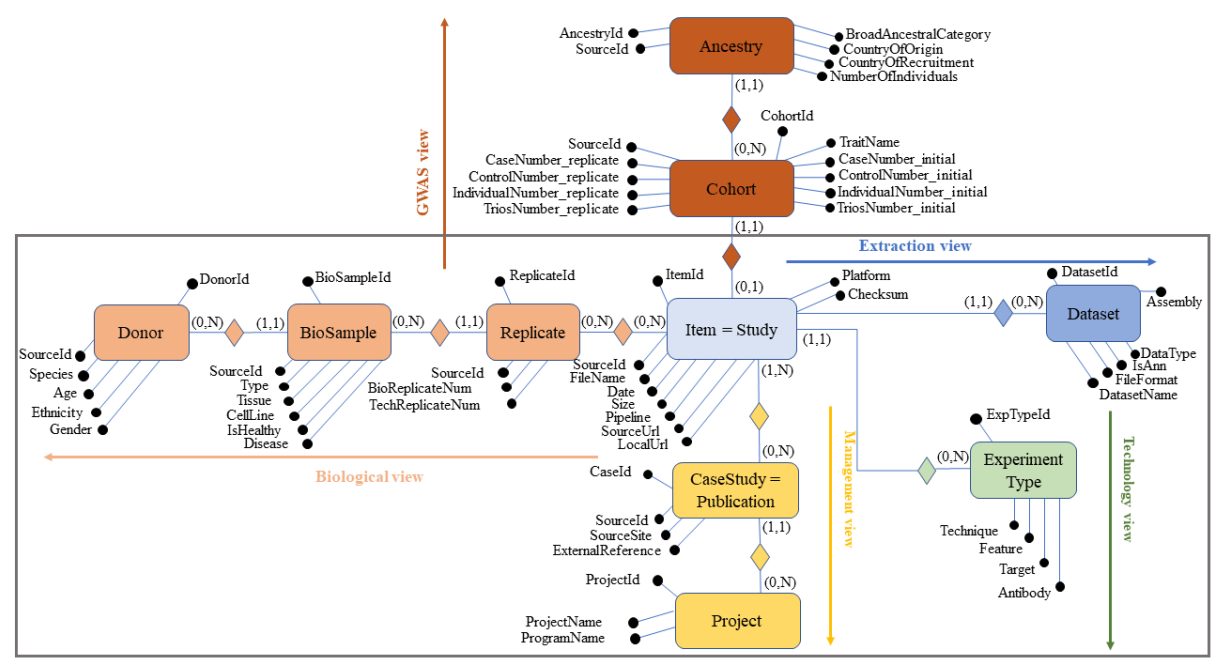

Fig. 1. Extended Genomic Conceptual Model. With respect to its original version (enclosed in the rectangle), it contains a new view (the GWAS View). When it gathers GWAS data, the Biological View remains empty (optional relationship between Item and Replicate); on the opposite when receiving different classes of studies, the GWAS View stays empty (optional relationship between Item and Cohort).

consideration. It contains metadata useful to describe how the corresponding region file (list of SNPs) is produced.

Entity Cohort. Each Item of the GCM has its corresponding Cohort which includes the information about the groups of people from which the biological sample is collected. An Item is obtained by comparing the DNA sequences of the cases (people affected by the phenotype) against the controls (people not showing that phenotype). Moreover, a sample can have one initial stage and one or more replicate stages. Some GWA studies, besides cases and controls, can be based upon groups of individuals or trios. The entity Cohort holds the cardinalities of the cases, controls, individuals or trios that provide the corresponding Item, both of the initial stage or replicate stage(s). Among all its metadata, the attribute "TraitName" is most relevant as GWA studies are driven by a phenotype (or trait, endpoint, disease).

Entity Ancestry. A Cohort can be partitioned into many Ancestries, each one containing given ancestral information about the current partition. The country of origin, the ancestral category or the country from which the participants are selected are possible available information about a partition.

Figure 2 shows how the source-specific metadata of GWAS Catalog and FinnGen are mapped into the attributes of the GCM: in the left column we list the attributes of the GCM, in the central column we report the attributes of 


\begin{tabular}{|c|c|c|}
\hline $\mathrm{GCM}$ & GWAS Catalog & FinnGen \\
\hline & Ancestry & \\
\hline broad_ancestral_category & broad_ancestral_category_x & -- \\
\hline country_of_origin & country_of_origin_X & - \\
\hline country_of_recruitment & country_of_recruitment_x & "Finland" \\
\hline number_of_individuals & number_of_individuals_x & n_cases + n_controls \\
\hline \multirow{2}{*}{ ancestry_source id } & study accession & phenocode \\
\hline & Cohort & \\
\hline trait_name & mapped_trait & name \\
\hline case_number_initial & initial_sample_description [MANUAL] & n_cases \\
\hline control_number_initial & initial_sample_description [MANUAL] & n_controls \\
\hline individual_number_initial & initial_sample_description [MANUAL] & -- \\
\hline triosNumber_initial & initial_sample_description [MANUAL] & - \\
\hline case_number_replicate & replication_sample_description [MANUAL] & - \\
\hline control_number_replicate & replication_sample_description [MANUAL] & -- \\
\hline individual_number_replicate & replication_sample_description [MANUAL] & -- \\
\hline trios_number_replicate & replication_sample_description [MANUAL] &.- \\
\hline \multirow[t]{2}{*}{ cohort_source_id } & study_accession & phenocode \\
\hline & Dataset & \\
\hline name & dataset_name & dataset_name \\
\hline data_type & "gwas" & "gwas" \\
\hline format & "gdm" & "gdm" \\
\hline assembly & "GRCh38" & "GRCh38" \\
\hline \multirow[t]{2}{*}{ is_annotation } & "false" & "false" \\
\hline & Item & \\
\hline item_source_id & study_accession & phenocode \\
\hline size & manually_curated_origin_file_size & manually_curated_local_file_size \\
\hline date & manually_curated_origin_last_modified_date & manually_curated_download_date \\
\hline checksum & manually_curated_origin_md5 & manually_curated_local_md5 \\
\hline platform & platform_snps_passing_qc_ & - \\
\hline \multirow[t]{2}{*}{ file_name } & study_accession + "gdm" & phenocode + "gdm" \\
\hline & Experiment_type & \\
\hline \multirow[t]{2}{*}{ technique } & genotyping_technology & "FinnGen_technique" \\
\hline & Case_study & \\
\hline case_source_id & pubmedid & phenocode \\
\hline source_site & study & "https://www.finngen.fi/en" \\
\hline \multirow[t]{2}{*}{ path_https } & link & externalRef \\
\hline & Project & \\
\hline program_name & "Gwas Catalog" & "FinnGen" \\
\hline project name & "Gwas Catalog" & "FinnGen" \\
\hline
\end{tabular}

Fig. 2. Attribute mapping from source-specific metadata to Genomic Conceptual Model. In this figure are not reported the items of the Biological View since it has no corresponding GWAS metadata.

GWAS Catalog, while in the right column we provide the attributes of FinnGen. The metadata enclosed by quotes are meant to be treated as values to fill the corresponding GCM attributes. Let us consider the metadata assembly; for both the two sources the value "GRCh38" is manually inserted since it is not contained in any of the raw attributes.

Some GCM attributes are obtained by the concatenation of two raw metadata (e.g., file_name $=$ study_accession + "gdm") or by their sum, if they are numeric (e.g., number_of_individuals $=n_{-}$cases $+n_{-}$controls $)$.

The progressive numbers nearby the attributes describing the ancestries of GWAS Catalog refer to the multiple ancestries linked to a single cohort. Let us consider the instance of the GCM proposed in Figure 3 , the item with accession "GCST007269" is linked to the cohort with id "2055", which is linked to two different ancestries (respectively ids "5473" and " 5476 "). The attributes of these 
two ancestries, before being mapped, are referred with two different progressive numbers.

\begin{tabular}{|c|c|c|c|c|c|c|c|c|c|}
\hline \multicolumn{4}{|c|}{ ancestry } & \multicolumn{3}{|c|}{ cohort } & \multicolumn{3}{|c|}{ item } \\
\hline id & cohort_id & \begin{tabular}{|l|} 
category \\
\end{tabular} & country & id & item_id & trait_name & item_id & file_name & dataset \\
\hline 5473 & 2055 & European & NR & 2055 & 2054 & pulse pressure & 2054 & GCST007269.gdm & 1 \\
\hline 5476 & 2055 & Native & U.S. & 2056 & 2055 & diabetes & 2055 & GCST009379.gdm & 1 \\
\hline 5480 & 2056 & \begin{tabular}{|l|} 
European \\
\end{tabular} & NR & 2057 & 2056 & \begin{tabular}{c|} 
membranous \\
glomerulonephritis
\end{tabular} & 2056 & GCST010004.gdm & 1 \\
\hline 5483 & 2057 & East Asian & $\begin{array}{l}\text { China } \\
\text { Turkey }\end{array}$ & 2058 & 2057 & $\begin{array}{c}\text { membranous } \\
\text { glomeruonephritis }\end{array}$ & 2057 & GCST010005.gdm & 1 \\
\hline $\begin{array}{l}5484 \\
5486\end{array}$ & $\begin{array}{l}2058 \\
2060\end{array}$ & \begin{tabular}{|c|} 
European \\
NR
\end{tabular} & \begin{tabular}{|c|} 
Turkey \\
Finland \\
\end{tabular} & 2060 & 2059 & viral fevers & 2059 & AB1_ARTHROPOD.gdm & 2 \\
\hline 5487 & 2061 & NR & Finland & 2061 & 2060 & infectious agents & 2060 & AB1_BACT_BIR.gdm & 2 \\
\hline 5488 & 2062 & NR & Finland & 2062 & 2061 & Helminthiases & 2061 & AB1_HELMINTIASES.gdm & 2 \\
\hline
\end{tabular}

Fig. 3. An instance of the Genomic Conceptual Model containing four items from GWAS Catalog (light blue) and three items from FinnGen (green). In this figure are reported only the entities corresponding to the GWAS View. Moreover, are reported only the relevant attributes to show the proper cardinalities between the entities, the full list of the attributes is reported in Figure 2

The attributes marked with the label "MANUAL" are not reported as they are but need a syntactic transformation. The metadata initial_sample_description and replication_sample_description are written in plain text: their values are parsed to fill the GCM attributes of the cohort. Here we report the example item extracted from study accession "GCST005538": initial_sample_description = 1,726 European ancestry cases, 5,482 European ancestry controls; replication_sample_description = 1,912 European ancestry cases, 5,938 European ancestry controls, 781 African American cases, 876 African American controls. As a result of our transformation, the attributes of the corresponding cohort become: CaseNumber_initial $=1726$, ControlNumber_initial $=5,482$, CaseNumber_replicate $=1,912+781$, ControlNumber_replicate $=5,938+876$.

\section{The case of "traitName"}

GWAS studies follow the phenotype-first approach: the participants of these studies are classified according to their clinical manifestations. The feature of GWAS studies is to search for SNPs given a phenotype. This is the reason why it is interesting to understand the set of phenotypes present in both the sources considered in this work.

All traits in GWAS Catalog are mapped over the EFO ontology [6]. Traits in the GWAS Catalog are highly diverse and include diseases (e.g., Type II diabetes), disease markers (e.g., measurements of blood glucose concentration), and non-clinical phenotypes (e.g., hair color). The Experimental Factor Ontology was chosen as the ontology to represent GWAS Catalog traits as it is highly adaptable and extensible. It is freely available in OWL format from the EFO 
website and can be browsed in the Ontology Lookup Service. At the moment of writing (July 2021), the GWAS Catalog contains 2,413 different traits from the EFO ontology. Each study is characterized by one or more traits contained into the source-specific attribute "mapped_trait", comma-separated.

FinnGen phenotypes are instead harmonized over the International Classification of Diseases (ICD) revisions 8, 9 and 10, cancer-specific ICD-O3, (NOMESCO) procedure codes, Finnish-specific Social Insurance Institute (KELA) drug reimbursement codes and ATC-codes [4. The latest release at the moment of writing (July 2021) is the fifth one. In its manifest all the files available are listed, each with its corresponding phenotype. The fifth release contains 2803 different phenotypes.

Applying exact string matching between the list of phenotypes of the two sources, 94 traits are found to be shared. More sophisticated semantic matches are subject to future extension of this work.
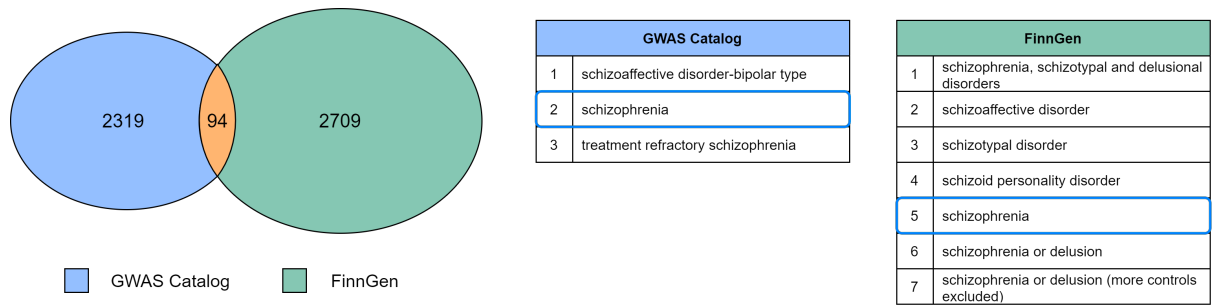

Fig. 4. Left: Intersection of the sets of phenotypes of the two sources GWAS Catalog and FinnGen. The intersection is obtained through exact matching of the two sets and it represents a small portion of both of them. Right: Traits related to "schizophrenia". The blue table reports the phenotypes of GWAS Catalog; the green table is dedicated to FinnGen. Only one trait is shared, all the others require domain experts to be correctly mapped.

A graphical representation of the intersection of the sets of phenotypes (with exact matching) is provided in Figure 4 . In the same figure, we report an example of the matching between the phenotypes of the two repositories. In both tables we report all the phenotypes resulting by searching for the word "schizophrenia". Only one common phenotype is spotted using exact matching; more correspondences may be found with semantic match (note that mapping phenotypes requires further effort and experts validation).

\section{Datasets interoperability}

Using the GenoMetric Query Language (GMQL) [10] many genomic datasets belonging to the GeCo repository can be jointly queried based on the values of some corresponding attributes. The GMQL operators exploit the transformed source-specific attributes and not the original ones, to ease the matching between 
attributes. The most significant attributes of GWA studies are the names of the phenotypes (attribute traitName of the cohort) and the ancestral information of the cohort upon which the studies are based on (attributes countryOfOrigin and countryOfRecruitment of the ancestry).

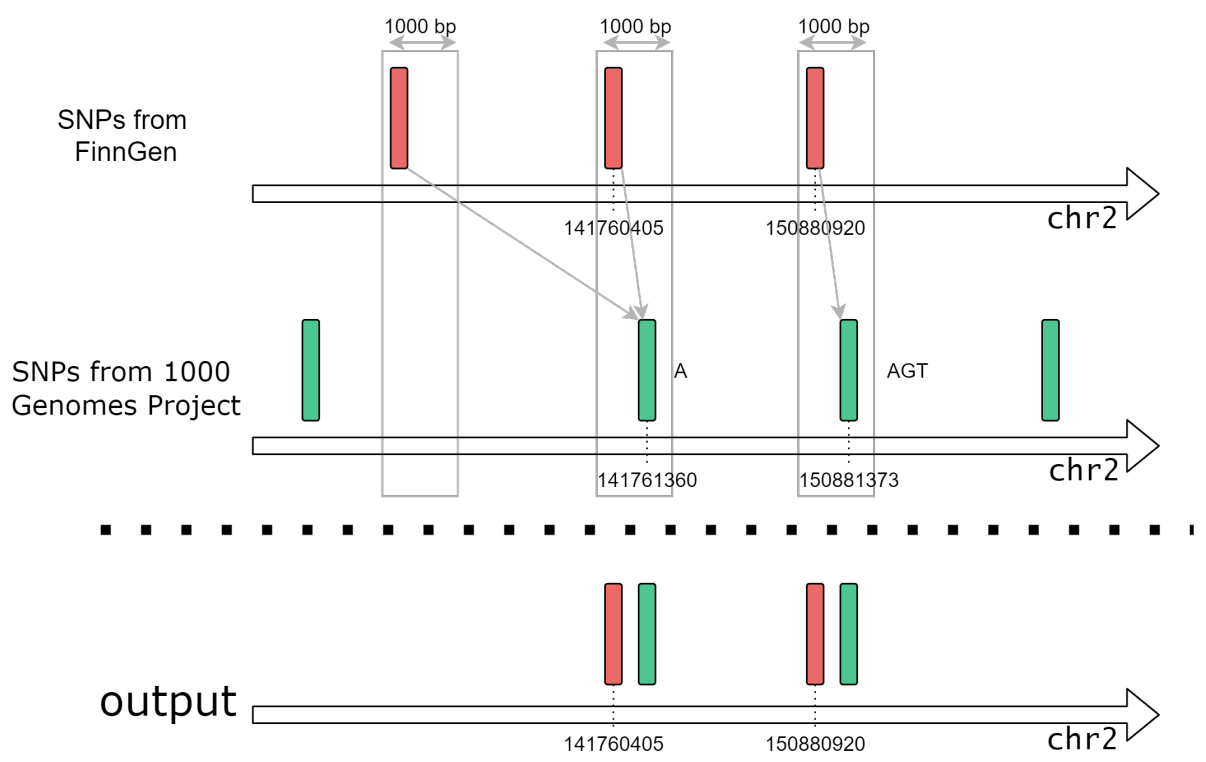

Fig. 5. GMQL query exploiting the integrated datasets FinnGen and 1000 Genomes Project. The query merges the two datasets to extract the couples made of SNPs that are closer than 1000 base pairs. It has been enabled by the META-BASE pipeline and the shared GCM.

Figure 5 graphically represents a query that jointly exploits the FinnGen dataset and the data from 1000 Genomes Project. The genomes sequenced in the 1000 Genomes Projects are not selected with regard to phenotype, so to provide a resource of variants that supports a deeper understanding of newly discovered loci influencing human disease. The projects include SNPs with allele frequencies as low as $1 \%$ across the genome and $0.1-0.5 \%$ in gene regions, as well as structural variants. It includes genomes from 26 different populations, including the Finnish one.

In the considered query, the SNPs related to the Finnish population are selected from 1000 Genomes, thereby enabling comparisons with the SNPs from FinnGen dataset. From this latter dataset we select only the SNPs which have been found as related to schizophrenia. The goal of the query is to analyze which SNPs of the two datasets are particularly close, specifically within an interval of 1000 base pairs. In the example, only two pairs of SNPs are eventually extracted in the output, as they meet the set distal constraint. Note that the querying 
of the two initial datasets was only enabled thanks to the integration efforts of metadata and region attributes introduced in this article and building up on [21].

\section{Conclusions}

GWAS studies are important because they allow to find numerous compelling associations for human complex traits and diseases. Once such genetic markers are identified, they can be used to understand how genes contribute to the diseases and to develop better prevention and treatment strategies. These associations have led to insights into the architecture of disease susceptibility (through the identification of novel disease-causing genes and mechanisms) and to advances in clinical care (for example, the identification of new drug targets and disease biomarkers) and personalized medicine (for example, risk prediction and optimization of therapies based on genotype).

The integration of GWAS with other classes of genomic data is fundamental to reach interoperability and answering complex biological questions. The exact interpretation of the SNPs found in GWAS is not trivial for at least two reasons. First, the outputs of GWAS are often large clusters of SNPs in linkage disequilibrium, making it difficult to distinguish causal SNPs from neutral variants in linkage. Second, even assuming the causal variants can be identified, interpretation is limited by incomplete knowledge of non-coding regulatory elements, their mechanisms of action and the cellular states and processes in which they function. For the aforementioned reasons, it is important to further investigate GWAS data by merging different genomic datasets and by performing multi-omic analyses.

We started from the GCM, which proposed an integrative schema solution for several genomic repositories. We extended it by adding three entities that are relevant for GWAS and we implemented the data import and transformation pipeline to store the datasets of two new data sources (i.e., GWAS Catalog and FinnGen) within the META-BASE repository. We detailed the mapping effort performed for this purpose and finally demonstrated the usefulness of this work by using a domain-specific language that interrogates FinnGen together with 1000 Genomes datasets for extracting SNPs relevant to the schizophrenia trait.

Acknowledgement. This research is funded by the ERC Advanced Grant 693174 GeCo (data-driven Genomic Computing).

\section{References}

1. Bernasconi, A., et al.: META-BASE: a novel architecture for large-scale genomic metadata integration. IEEE/ACM Transactions on Computational Biology and Bioinformatics (2020)

2. Bernasconi, A., et al.: Conceptual modeling for genomics: building an integrated repository of open data. In: Int. Conf. on Conceptual Modeling. pp. 325-339. Springer (2017) 
3. Buniello, A., et al.: The NHGRI-EBI GWAS Catalog of published genome-wide association studies, targeted arrays and summary statistics 2019. Nucleic acids research 47(D1), D1005-D1012 (2019)

4. FinnGen Project: 5th release. https://finngen.gitbook.io/documentation/ (2020)

5. Frankish, A., et al.: GENCODE reference annotation for the human and mouse genomes. Nucleic acids research 47(D1), D766-D773 (2019)

6. GWAS Catalog team: GWAS catalog website. https://www.ebi.ac.uk/gwas/

7. International Multiple Sclerosis Genetics Consortium: Multiple sclerosis genomic map implicates peripheral immune cells and microglia in susceptibility. Science 365(6460) (2019)

8. Kundaje, A., et al.: Integrative analysis of 111 reference human epigenomes. Nature $\mathbf{5 1 8}(7539), 317-330$ (2015)

9. Masseroli, M., et al.: Modeling and interoperability of heterogeneous genomic big data for integrative processing and querying. Methods 111, 3-11 (2016)

10. Masseroli, M., et al.: GenoMetric Query Language: a novel approach to large-scale genomic data management. Bioinformatics 31(12), 1881-1888 (2015)

11. Palacio, A.L., et al.: A method to identify relevant genome data: conceptual modeling for the medicine of precision. In: Int. Conf. on Conceptual Modeling. pp. 597-609. Springer (2018)

12. Pastor, O.: Understanding the human genome: a conceptual modeling-based approach. In: Int. Conf. on Database and Expert Systems Applications. pp. 467-469. Springer (2010)

13. Pastor, O., et al.: Enforcing conceptual modeling to improve the understanding of human genome. In: 2010 Fourth Int. Conf. on Research Challenges in Information Science. pp. 85-92. IEEE (2010)

14. Rambold, G., et al.: Meta-omics data and collection objects (MOD-CO): a conceptual schema and data model for processing sample data in meta-omics research. Database 2019

15. Román, J.F.R., et al.: Applying conceptual modeling to better understand the human genome. In: Int. Conf. on Conceptual Modeling. pp. 404-412. Springer (2016)

16. Rudy, G., Helix, G.: A hitchhiker's guide to next-generation sequencing. http: //www.goldenhelix.com/pdfs/whitepapers/Hitchhikers-Guide-to-NGS.pdf (2010)

17. Schuster, S.C.: Next-generation sequencing transforms today's biology. Nature methods 5(1), 16-18 (2008)

18. Tam, V., et al.: Benefits and limitations of genome-wide association studies. Nature Reviews Genetics 20(467-484) (2019)

19. The 1000 Genomes Project Consortium: A map of human genome variation from population-scale sequencing. Nature 467(7319), 1061-1073 (2010)

20. The ENCODE Project Consortium: An integrated encyclopedia of DNA elements in the human genome. Nature $\mathbf{4 8 9}$ (7414), 57-74 (2012)

21. Wang, K., et al.: A genome-wide meta-analysis identifies novel loci associated with schizophrenia and bipolar disorder. Schizophrenia Research 124(1), 192-199 (2010)

22. Wang, L., et al.: Biostar models of clinical and genomic data for biomedical data warehouse design. Int. j. of bioinform. research and applications 1(1), 63-80 (2005)

23. Weinstein, J.N., et al.: The cancer genome atlas pan-cancer analysis project. Nature genetics 45(10), 1113-1120 (2013)

24. Zheng, R., et al.: Cistrome Data Browser: expanded datasets and new tools for gene regulatory analysis. Nucleic Acids Research 47(D1), D729-D735 (2018) 